\title{
Study of the Physical Properties of Cobalt/ Cobalt Oxide Particles Synthesized by Pulsed Laser Ablation in Different Liquid Media
}

\author{
S.M. Borghei* AND F. BAKHTIYARI \\ Department of Physics, Karaj Branch, Islamic Azad University, Karaj, Iran
}

\begin{abstract}
This work is devoted to the production of microparticles via pulsed laser ablation from a solid cobalt target immersed in three different solutions of distilled water as well as in ethanol and in acetone, to study the effect of liquid media on the physical properties and structure of particles. The samples were characterized using XRD, SEM and particle size analysis. More than simply Co particles have resulted in each three liquid media, CoO particles were produced only in acetone, whereas $\mathrm{Co}_{3} \mathrm{O}_{4}$ particles were predominantly produced in water and ethanol.
\end{abstract}

DOI: 10.12693/APhysPolA.131.332

PACS/topics: 81.07.-b, 75.75.Cd, 52.38.Mf

\section{Introduction}

For the first time, high power laser was used to synthesize iron oxide at a liquid-solid surface in 1987 [1]. After that many researchers have used this method to produce other magnetic transition metals and metal oxide particles, such as cobalt or nickel and have opened the doors of new challenges for the researches. Laser ablation theory and problems therein are very important and theoretical part has been also investigated along with experimental works [2].

In the last two decades, different methods have been developed for controlling the size, phase, shape and other features of the particles. These include diverse physical or chemical methods [3-7]. Among these methods, laser ablation technique has been widely used for producing metal and metal oxide micro and nanostructures. A significant advantage of this method is the possibility to prepare noble metal particles. It is also applicable for production of compound materials such as $\mathrm{TiO}_{2}, \mathrm{ZnSe}$ and $\mathrm{CdS}[8,9]$.

It has been also found that the type of liquid medium and the target have a significant impact on the properties of produced particles. This approach produces particles which are usually spherical or quasi-spherical. However, other structures such as hollow particles, cubes, rods, cones, discs, plates, sheets and other complex structures are observed. In the case of non-spherical particles, crystal structure and surface active substances in liquids can severely affect the morphology.

Magnetic metal/metal oxide particles have various fundamental and industrial applications. Cobalt is a special element that changes between two phase structures of hcp and fcc at a transition temperature of $450{ }^{\circ} \mathrm{C}$ [10]. It is

*corresponding author; e-mail: majid.borghei@kiau.ac.ir a ferromagnetic material, and due to such interesting properties, as good mechanical hardness, chemical stability and high electrical resistance, it has been used in many applications, including magnetic photo catalysts, high frequency magnets, information storage systems, magnetic bulk cores, microwave absorbers and in biological applications, such as drug delivery, magnetic resonance imaging and bio-sensors [11, 12].

Cobalt oxides have three oxidation states, namely: (i) cobaltous oxide (cobalt monoxide or cobalt [II] oxide) with $\mathrm{CoO}$ chemical formula, (ii) cobaltic oxide (cobalt [III] oxide) with $\mathrm{Co}_{2} \mathrm{O}_{3}$ chemical formula and (iii) cobaltosic oxide (cobalt [II, III] oxide) with $\mathrm{Co}_{3} \mathrm{O}_{4}$ chemical formula, which is an antiferromagnetic material. The first and the last oxidation states have a cubic crystal structure. In general, these oxidation states allow fundamental applications in gas sensors and electrochromic devices, in chemical and ceramic industries, in glass and porcelain industries, as an additive coloring agent, in superconductors, etc.

Previously, laser ablation of cobalt and its oxides in liquids has been reported by $\mathrm{T}$. Tsuji et al. [13]. They have used $\mathrm{Co}, \mathrm{CoO}$ and $\mathrm{Co}_{3} \mathrm{O}_{4}$ powders suspended in water/hexane. Here we have used ablation of a cobalt solid target, implementing a pulsed laser to synthesis in liquids (PLASIL). In other words, cobalt and cobalt oxide particles are produced by one target immersed in three different media: water, ethanol and acetone. The synthesis was carried out to investigate the influence of liquid medium on the production of resulted $\mathrm{Co}, \mathrm{CoO}$ and $\mathrm{Co}_{3} \mathrm{O}_{4}$ particles. Finally, the samples were characterized by SEM, particle size analysis (PSA) and XRD.

The paper is organized as follows. After a short introduction, most important properties of Co and its oxides along with applications are briefly reviewed. In the next section, the experimental set up and ablation conditions are presented. The results of sample characterization and discussions are given in the third section. Finally, conclusions are summarized in last section. 


\section{Experimental section}

\subsection{Experimental setup and conditions}

A solid cobalt target $(75 \mathrm{~g})$, was placed at the bottom of a glass beaker filled with $15 \mathrm{ml}$ of water, ethanol or acetone. It was ablated for 10 min using a Nd:YAG laser light $(1064 \mathrm{~nm})$, repetition rate of $3 \mathrm{kHz}$, at a laser fluency of $3 \times 10^{4} \mathrm{~J} / \mathrm{cm}^{2}$, with pulse duration of $150 \mathrm{~ns}$. Laser light was focused by a lens $(f=160 \mathrm{~mm})$. The setup employed in this work is illustrated in Fig. 1.

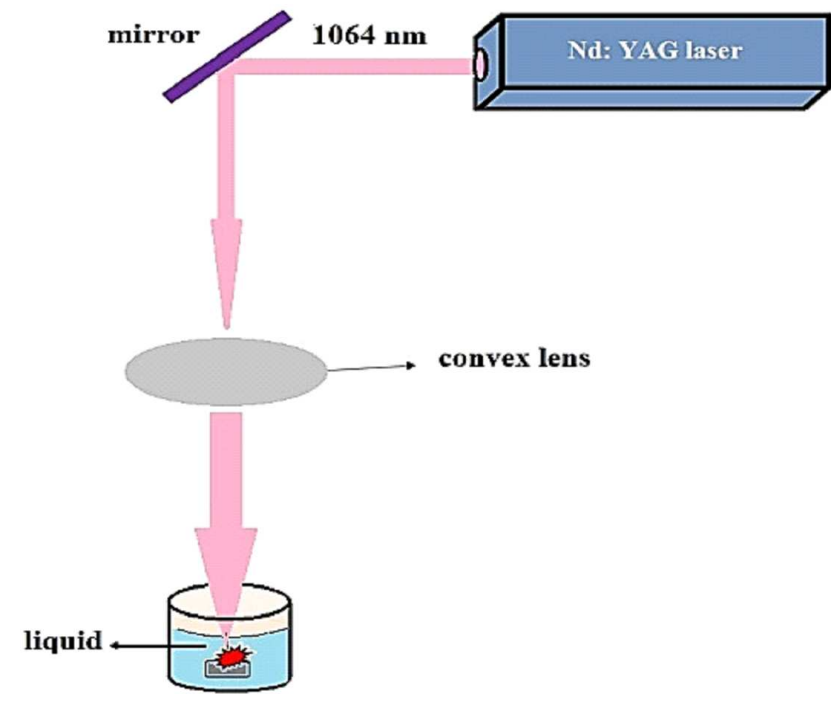

Fig. 1. Experimental setup used for PLASIL.

\subsection{Characterizations}

Cobalt/cobalt oxide particles were characterized using a SEM operated at $25 \mathrm{kV}$. Particle size analysis was carried out to estimate their average sizes and size distribution. XRD was also conducted using a Philips PW 3710 model with $2 \theta$ range from $10^{\circ}$ to $70^{\circ}$ in $0.5^{\circ} \mathrm{min}^{-1}$ steps.

\section{Results and discussion}

To illustrate the effect of laser environment on the characteristics of cobalt and cobalt oxide particles, produced in three different media (acetone, ethanol and distilled water), scanning electron microscopy was employed. SEM images of particles prepared in these media are shown in Fig. 2. The morphology of the particles can be observed in the figure. As we can see, the particles are generally spherical, but some of them are sticking together and are agglomerated, resulting in a larger mass of quasispherical shape, such as those, which we can obviously see for acetone and distilled water. In the case of acetone, most of the particles sizes are nearly one micrometer, whereas there are some larger particles. To prepare samples for SEM microscopy, the colloids containing cobalt and cobalt oxides were dried on a silicon substrate. Sizes of the particles were measured based on the microscopy images. As the colloidal particles were dried more than ten times, the results of measurements cannot be exact. It must be also noted that microscope images show only very small areas on the surface and microscopy in another place may results in different results.

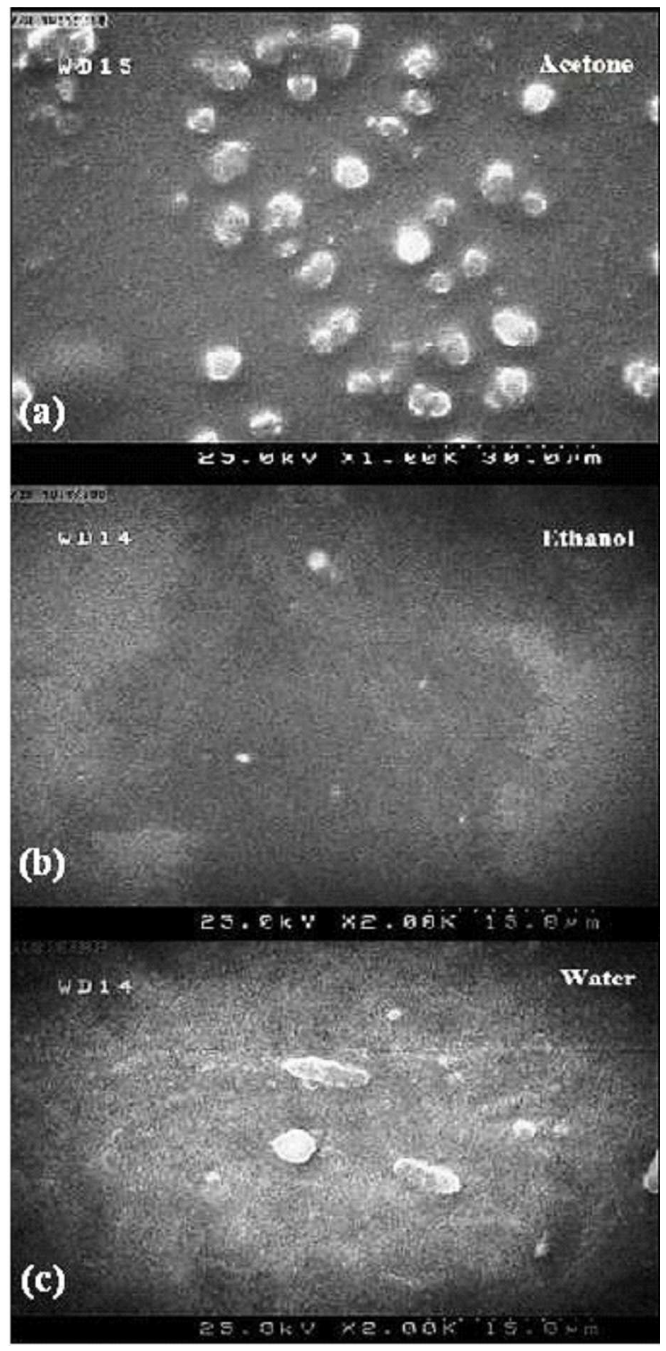

Fig. 2. SEM images of particles, prepared by laser ablation in (a) acetone, (b) ethanol and (c) distilled water, dried on the silicon substrate surface.

To have a better insight into the size and its distribution of as-ablated particles in the colloid, PSA was also performed and results are shown in Fig. 3.

The size and distribution of the cobalt/cobalt oxide colloidal particles related to each environmental ablation medium is clear. It can be seen that by changing the liquid solution, from distilled water and ethanol to acetone, the particles size decreases. Particles produced in distilled water and ethanol are in the range of 1 to nearly 20 micrometers and become smaller 0.2 to 2 micrometers by changing the solution to acetone.

In Fig. 4, we can see X-ray diffraction measurements that were performed for the dried particles on silicon substrate, by scanning in the $2 \theta$ angle range of $10-70^{\circ}$. 

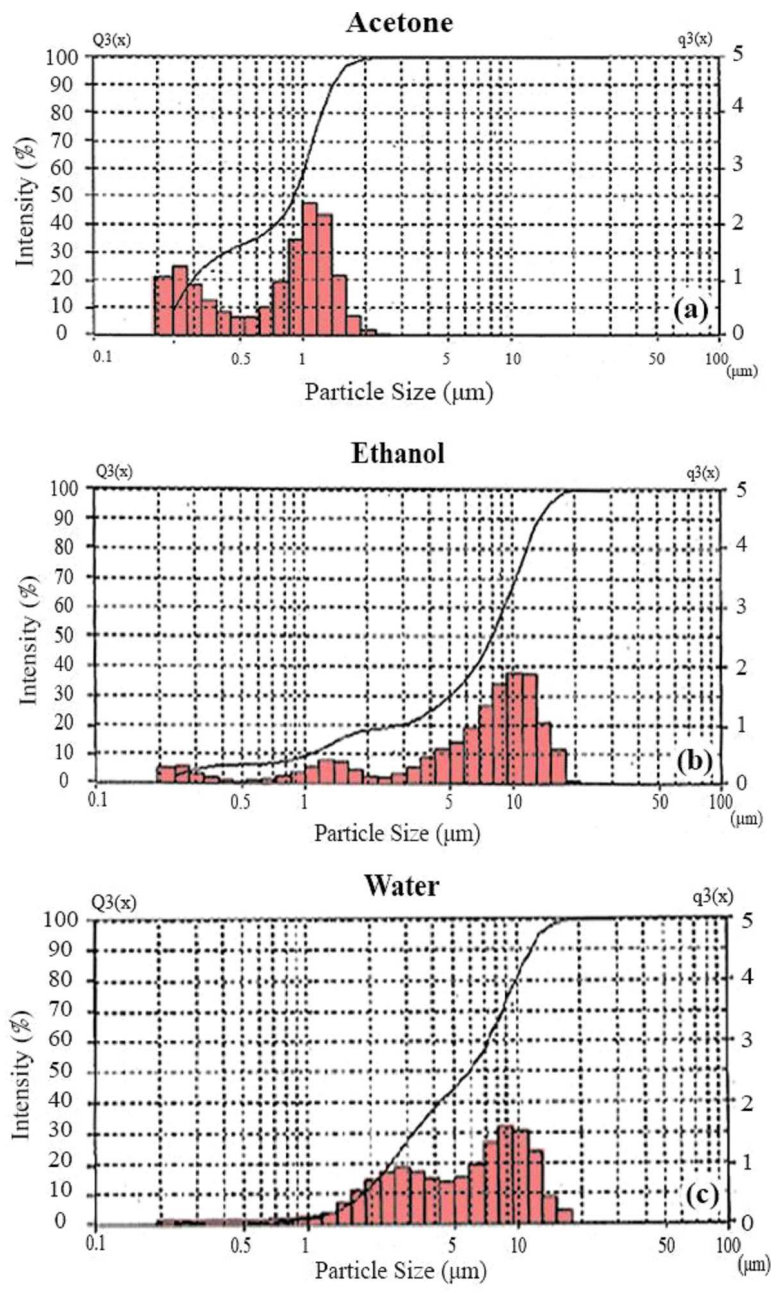

Fig. 3. PSA analysis of cobalt colloidal particles produced in three liquid media: (a) acetone, (b) ethanol and (c) distilled water.

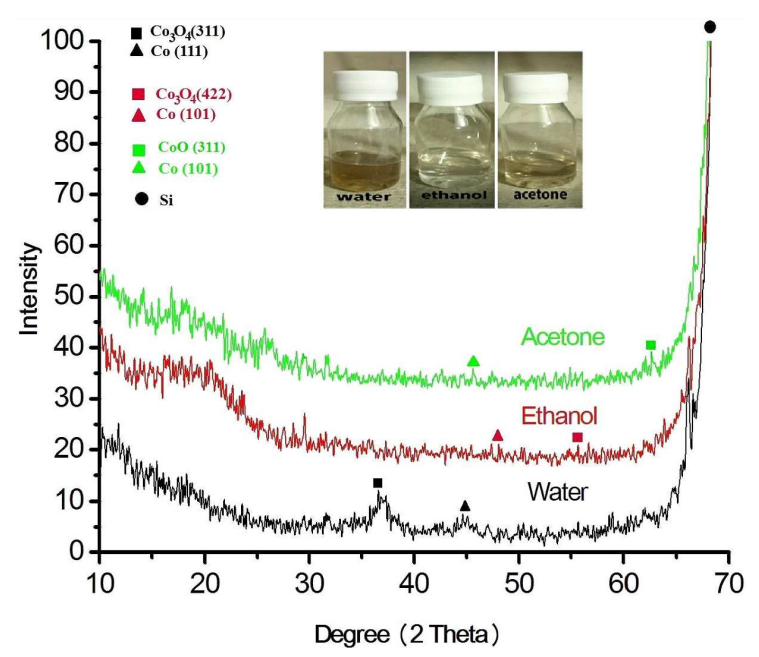

Fig. 4. X-ray diffraction of cobalt and its oxide particles, prepared by laser ablation mechanism in acetone, ethanol and distilled water.
The formation of cobalt oxide particles is confirmed and the XRD spectra, clearly show the crystalline structure of the various peaks of cobalt and cobalt oxide particles.

The XRD pattern of particles formed by $1064 \mathrm{~nm}$ wavelength laser beam in distilled water at room temperature reveals that particles are crystalline and contain peaks at $2 \theta=36.5^{\circ}$ and $2 \theta=44.5^{\circ}$, which belong to cubic structures of $\mathrm{Co}_{3} \mathrm{O}_{4}$ (311) and $\mathrm{Co}$ (111) respectively. The average crystallite size can be deduced considering Scherer formula [14]. According to the full width at half maximum of the diffraction peaks, one can conclude that this size varies from one liquid medium to another. For particles prepared in ethanol liquid medium, it can be observed that the peaks at angles of $2 \theta=47.3^{\circ}$ and $2 \theta=55.7^{\circ}$ are related to hexagonal structure of Co (101) and cubic structure of $\mathrm{Co}_{3} \mathrm{O}_{4}(422)$. Finally, for acetone the peaks of $2 \theta=47.3^{\circ}$ and $2 \theta=62.7^{\circ}$ give the hexagonal structure of $\mathrm{Co}$ (101) and cubic structure of $\mathrm{CoO}$ (311). The inset shows the cobalt/cobalt oxides particles in each medium. The color of particle suspension in water is light brown, whereas in ethanol it is colorless and in acetone it is light yellow.

\section{Conclusions}

In this study, the effect of laser environment on such characteristics of cobalt/cobalt oxide particles as morphology, size and size distribution, phase and structure was investigated. Cobalt and three states of cobalt oxide particles were successfully prepared by PLASIL on a cobalt target in acetone, ethanol and distilled water, in a glass beaker. SEM images show that the generated particles are spherical and quasi-spherical. In general, it can be concluded that the interaction of laser beam light with target in distilled water and ethanol results in production of larger particles compared to those in acetone environment. In other words, acetone gives a smaller class of microparticles. However, XRD analysis shows a better crystalline structure of the particles produced in water compared to those prepared in ethanol and acetone. These results indicate that the choice of solution is very important to control the size properties, composition, phase and crystallinity of the particles produced by laser ablation in liquids.

\section{Acknowledgments}

This work has been fully supported by the Karaj Branch of the Islamic Azad University. The authors also acknowledge Mr. Moghri Mo'azzen and Mr. Azmoodeh for their kind help, preparation of the setup and facilitating the experiments.

\section{References}

[1] P. Patil, D.M. Phase, S.A. Kulkarni, S.V. Ghaisas, S.K. Kulkarni, S.M. Kanetkar, S.B. Ogale, V.G. Bhide, Phys. Rev. Lett. 58, 238 (1987).

[2] S.I. Anisimov, B.S. Luk'yanchuk, Physics-Uspekhi. 45, 293 (2002). 
[3] W. Estrada, M.C.A. Fantini, S.C. de Castro, C.N. Polo da Fonseca, A. Gorenstein, J. Appl. Phys. 74, 5835 (1993).

[4] A. Sarikas, E. Canayaz, U. Kesen, in: 2nd Int. Symp. Innovative Technologies in Engineering and Science (ISITES 2014), Karabuk, Turkey 2014, p. 715.

[5] U. Kesen, T. Yildirim, in: Proc. 15th Int. Symp. Computer and Information Sciences (ISCIS XV), Istanbul, Turkey 2000, p. 545.

[6] J. Vidal-Vidal, J. Rivas, M.A. Lopez-Quintela, Coll. Surf. A: Phys. Eng. Asp. 288, 44 (2006).

[7] F. Svegl, B. Orel, I Grabec-Svegl, V. Kaucic, Electrochem. Acta 45, 4359 (2000).

[8] M. Sugiyama, H. Okazaki, S. Koda, Jpn. J. Appl. Phys. 41, 4666 (2002).
[9] K.V. Ankin, N.N. Melnik, A.V. Simakin, G.A. Shafeev, V.V. Voronov, A.G. Vitukhnovsky, Chem. Phys. Lett. 366, 357 (2002).

[10] M. Jiang, K. Oikawa, T. Ikeshoji, Metallur. Mater. Trans. A 36, 2307 (2005).

[11] P. Razmjouei, S.M. Mirkazemi, M. Jalalian, J. Ir. Cer. Soc. 32-33, 63 (2012) (in Persian).

[12] B.K. Pandey, A.K. Shahi, R. Gupal, Appl. Surf. Sci. 347, 461 (2015).

[13] T. Tsuji, T. Hamagami, T. Kawamura, J. Yamaki, M. Tsuji, Appl. Surf. Sci. 243, 214 (2005).

[14] B.D. Cullity, Elements of X-Ray Diffraction, Addison-Wesley Pub., 2005. 